\title{
Orphan nuclear receptor NR4A2 inhibits hepatic stellate cell proliferation through MAPK pathway in liver fibrosis
}

Pengguo Chen, Jie Li, Yan Huo, Jin Lu, Lili Wan, Bin Li, Run Gan, Cheng Guo

Hepatic stellate cells (HSCs) play a crucial role in liver fibrosis, which is a pathological process characterized by extracellular matrix accumulation. NR4A2 is a nuclear receptor belonging to the NR4A subfamily and vital in regulating cell growth, metabolism, inflammation and other biological functions. However, its role in HSCs is unclear. We analyzed NR4A2 expression in fibrotic liver and stimulated HSCs compared with control group and studied the influence on cell proliferation, cell cycle, cell apoptosis and MAPK pathway after NR4A2 knockdown. NR4A2 expression was examined by real-time polymerase chain reaction, Western blotting, immunohistochemistry and immunofluorescence analyses. NR4A2 expression was significantly lower in fibrotic liver tissues and PDGF BB or TGF- $\beta$ stimulated HSCs compared with control group. After NR4A2 knockdown $\alpha$-smooth muscle actin and Col1 expression increased. In addition, NR4A2 silencing led to the promotion of cell proliferation, increase of cell percentage in $\mathrm{S}$ phase and reduced phosphorylation of ERK1/2, P38 and JNK in HSCs. These results indicate that NR4A2 can inhibit HSC proliferation through MAPK pathway and decrease extracellular matrix in liver fibrogenesis. NR4A2 may be a promising therapeutic target for liver fibrosis. 


\section{Orphan nuclear receptor NR4A2 inhibits hepatic stellate cell proliferation 2 through MAPK pathway in liver fibrosis}

4 Pengguo Chen ${ }^{1,2}, \mathrm{Jie}_{\mathrm{Li}^{1}}$, Yan Huo ${ }^{1}$, Jin Lu ${ }^{1}, \mathrm{Lili}$ Wan ${ }^{1}, \mathrm{Bin} \mathrm{Li}^{1}$, Run Gan ${ }^{1}$ and Cheng Guo ${ }^{1,2 *}$ 5

$6{ }^{1}$ Department of Pharmacy, Shanghai Jiao Tong University Affiliated Sixth People's Hospital, 600 Yishan

7 Road, Shanghai, China

$8{ }^{2}$ Shanghai Jiao Tong University School of Medicine, Shanghai, China

9 *Corresponding author: Department of Pharmacy, Shanghai Jiao Tong University Affiliated Sixth People's

10 Hospital, 600 Yishan Road, Shanghai 200233, China. Fax: +86 02124058789

11 Email adress: guopharm@126.com (C.Guo)

\section{ABSTRACT}

Hepatic stellate cells (HSCs) play a crucial role in liver fibrosis, which is a pathological process characterized by extracellular matrix accumulation. NR4A2 is a nuclear receptor belonging to the NR4A subfamily and vital in regulating cell growth, metabolism, inflammation and other biological functions. However, its role in HSCs is unclear. We analyzed NR4A2 expression in fibrotic liver and stimulated HSCs compared with control group and studied the influence on cell proliferation, cell cycle, cell apoptosis and MAPK pathway after NR4A2 knockdown. NR4A2 expression was examined by real-time polymerase chain reaction, Western blotting, immunohistochemistry and immunofluorescence analyses. NR4A2 expression was significantly

22 lower in fibrotic liver tissues and PDGF BB or TGF- $\beta$ stimulated HSCs compared with control 
23 group. After NR4A2 knockdown $\alpha$-smooth muscle actin and Coll expression increased. In

24 addition, NR4A2 silencing led to the promotion of cell proliferation, increase of cell percentage

25 in S phase and reduced phosphorylation of ERK1/2, P38 and JNK in HSCs. These results

26 indicate that NR4A2 can inhibit HSC proliferation through MAPK pathway and decrease

27 extracellular matrix in liver fibrogenesis. NR4A2 may be a promising therapeutic target for liver

28 fibrosis.

29 Keywords Fibrosis Hepatic stellate cell MAPK NR4A2 Proliferation

\section{INTRODUCTION}

Liver fibrosis is a pathological process characterized by accumulation of extracellular matrix. It may be triggered by inflammation, drug, alcohol, virus and cholestasis (Cassiman et al., 2002).

A variety of cells such as endothelial cells, hepatic stellate cells (HSCs) and Kupffer cells are involved in liver fibrogenesis. Among them HSCs play a crucial role and are the main cell source of extracellular matrix (Reynaert et al., 2002). HSCs, also known as Ito cells or perisinusoidal cells, are pericytes found in the perisinusoidal space of the liver. When the liver is damaged, quiescent HSCs are activated.

MAPK/extracellular regulated kinase (ERK) pathway is vital for the activation of HSCs.

Recently liver fibrosis was found to be reversible which was demonstrated in animal models

(Cao et al., 2004; Saxena et al., 2007). However, some mechanisms are still unknown.

There are increasing studies on NR4A subfamily. NR4A members are widely distributed in cells mediating differentiation, proliferation and apoptosis and are involved in many diseases 
44 such as cancer, vascular sclerosis and metabolic syndrome. NR4A members share highly 45 conserved cellular region: a variable amino terminal area, a DNA-binding area at the center and a 46 variable and continuous D-area that connects DBD to carboxyl terminal conservative E/F area.

47 NR4A members are early phase reaction genes and serve as transcription factors (Rius et al., 48 2006; Zhao, Desai \& Zeng., 2011; Holla et al., 2011). Palumbo-Zerr et al observed that NR4A1 recruits a repressor complex limiting profibrotic TGF- $\beta$ effects. Persistent activation of TGF- $\beta$ suppresses NR4A1 expression in fibrotic diseases (Palumbo-Zerr et al., 2015). Yin et al discovered that expression of NA4A1, NR4A2 and NR4A3 decrease apparently in uterine fibroids (Yinet al., 2013). Studies on NR4A in liver disease are scarce. It is well confirmed that downregulation of ERK1 in HSCs remarkably attenuated the extracellular matrix deposition in fibrotic liver (Zhong et al., 2009). ERK5/MAPK in combination with NR4A2 results in boosting of transcriptional activity (Sacchett et al., 2006). In addition, NR4A2 is identified as the target of ERK2 (Zhang et al., 2007). Taken together, NR4A2 may modulate HSCs in liver fibrosis. tissues and PDGF BB or TGF- $\beta$ stimulated HSCs. NR4A2 silencing led to higher expression be a potential target for anti-fibrotic treatment.

MATERIALS \&METHODS 
65 Antibodies against glyceral-dehydephosphate dehydrogenase (GAPDH) (SC-25778), NR4A1

66 (SC-5569), NR4A2 (SC-991), NR4A3 (SC-30154) and a -SMA (SC-32251) were purchased 67 from Santa Cruz (CA, USA). Alexa Fluor ${ }^{\circledR} 546$ IgG antibody (A10040) and Alexa Fluor ${ }^{\circledR} 488$

68 IgG $(\mathrm{H}+\mathrm{L})$ antibody (A21202) were obtained from Life Technologies. IRDye680 anti-rabbit antibody (926-32221) and IRDye680 anti-mouse antibody (926-32220) were from LI-COR (NE, USA). Antibody against Erk1/2 (4695P), P-erk1/2 (4370P), JNK (9258P), P-JNK (4668P), P-P38 (4511P) and P38 (8690P) were purchased from Cell Signaling (MA, USA). Nycodenz (QK1002424-1) was from Nycomed (Zurich, Switzerland). NC nitrocellulose was from Pall (NK, U-SA). Deoxyribonuclease I was obtained from Roche (Basel, Switzerland). D-HanK' s and 30\% acrylamide solution were from Bio-Light (Shanghai, China). Diaminobenzidine (DAB) Kit (GK50705) was from GeneTech (Shanghai, China). Cell cycle and Apoptosis Analysis Kit were purchased from R\&S (Shanghai, China). Annexin V-FITC Apoptosis Detection Kit was from Keygen (NJ, China). TGF- $\beta 1$ was obtained from Peprotech (NJ, USA). Tween-20 and ammonium persulfate were obtained from Sinopharm (Beijing, China). Lipofect-amineTM 2000 was purchased from Invitrogen (CA, USA). Collagenase IV, PDGF BB and Pronase were purchased from Sigma (St. Louis, MO, USA). Fecal calf serum (FBS) was purchased from Gibco (NY, USA). RIPA buffer, phenylmethylsulfonyl fluoride, TEMED, 5× SDS loading buffer and BCA kit were from Beyotime (Shanghai, China).

\section{Animals and liver samples}

Male Sprague-Dawley rats (SLAC, Shanghai, China), weighing approximately 250-270 g were maintained in a 12-h dark/12-h light cycle. All animal experiments were approved by the Animal 
86 Care and Use Committee of Shanghai Jiao Tong University Affiliated Sixth People's Hospital

87 (License No. SYXK2011-0128). Human liver samples were offered by hepatobiliary surgery department of Shanghai Jiao Tong University Affiliated Sixth People's Hospital. All samples were obtained in accordance with the ethics committee of Shanghai Jiao Tong University.

\section{Cell culture}

The HSC-T6 cells were kindly provided by Dr. Friedman of Mount Sinai School of Medicine of New York University (MSSM). Cells were cultured in DMEM medium supplemented with 10\% heat-inactivated fetal calf serum (FBS). All cells were maintained in a humidified incubator at $37^{\circ} \mathrm{C}$ with $5 \% \mathrm{CO}_{2}$. Platelet-derived growth factor (PDGF) $\mathrm{BB}$ or TGF- $\beta$ was used for stimulation.

\section{RNA isolation and quantitative real time-polymerase chain reaction}

Total RNA was extracted from HSC-T6 cell using Trizol (Takara, Dalian, China) and re verse transcribed using PrimeScript RT Master Mix (Takara). Real time polymerase chain reaction (RT-PCR) was performed using SYBR Green PCR Kit (Takara) and Applied Bi osystems 7500 real-time PCR system (Applied Biosystems, Foster City, CA, USA). Prime r sequences (Sangon Biotech, Shanghai, China) were as follows: NR4A1 (Fw: 5'-ACACC GGAGAGTTTGACACC-3'; Rev： 5'-GGGTAGCAGCCATACACCTG-3'); NR4A2 (Fw：5' -AGATTCCTGGCTTTGCTGAC-3'; Rev： 5'-CTGGGTTGGACCTGTATGCT-3'); NR4A3 ( Fw: 5'-GGCTGCAAGGGCTTCTTCA-3'; Rev： 5'-CACCATCCCGACACTGAGACA); $\alpha-S$ 
107

108

109

110

111

112 113 as measured by the $2^{-\Delta \Delta C T}$ method.
115

116 117 118 119 120

MA (Fw: 5'-CCAGGGAGTGATGGTTGGA-3'; Rev:5'-CCGTTAGCAAGGTCGGATG-3'); Coll (Fw: 5'-AGGCATAAAGGGTCATCGTG-3'; Rev: 5'-ACCGTTGAGTCCATCTTTGC3'); $\beta$-actin (Fw: 5'-ACCCACACTGTGCCCATCTATG-3'; Rev: 5'-AGAGTACTTGCGCTC

AGGAGGA-3'). The cycle conditions were $95^{\circ} \mathrm{C}$ for $30 \mathrm{~s}, 40$ cycles at $95^{\circ} \mathrm{C}$ for $5 \mathrm{~s}$ and $60^{\circ} \mathrm{C}$ for $34 \mathrm{~s}$, one cycle at $95^{\circ} \mathrm{C}$ for $15 \mathrm{~s}, 60^{\circ} \mathrm{C}$ for $1 \mathrm{~min}$ and $95^{\circ} \mathrm{C}$ for $15 \mathrm{~s}$. The resulting se quences were normalized to the $\beta$-actin expression levels, and relative gene expression $\mathrm{w}$

\section{Immunohistochemistry and immunofluorescence}

Hepatic tissues were embedded in paraffin and sectioned. The sections were incubated in $3 \%$ $\mathrm{H}_{2} \mathrm{O}_{2}$ in methanol and nonspecific binding was blocked with $10 \%$ normal goat serum. The sections were incubated with primary antibody at $4{ }^{\circ} \mathrm{C}$ overnight, washed and incubated with secondary antibody for $60 \mathrm{~min}$ at room temperature. Antigen-antibody complexes were visualized using DAB kits.

Immunofluorescence was performed as described previously (García-Pérez et al., 2013; Garcia-Pérez et al., 2015). For detecting NR4A2 and $\alpha$-smooth muscle actin ( $\alpha$-SMA) expression in liver tissue, the sections were incubated for $48 \mathrm{~h}$ at $4{ }^{\circ} \mathrm{C}$ with the following primary antibodies: NR4A2 (1:50), Alexa Fluor ${ }^{\circledR} 546 \operatorname{IgG}(1: 500)$, Alexa Fluor ${ }^{\circledR} 488$ IgG $(\mathrm{H}+\mathrm{L})$ (1:500). The sections were incubated in 4, 6-diamino-2-phenylindole (DAPI, 1:100) for $1 \mathrm{~min}$ and mounted in antifade reagent. 
128

129

130

131

132

133

134

135

136

137

138

139

140

141

142

143

144

145

146

147

148

\section{NR4A2 siRNA transfection}

For RNA interference assay, the cells were seeded into 6-well cell culture plates at a de nsity of $5 \times 10^{5}$ cells. The cells were transfected with siRNAs and Lipofectamine 2000 a ccording to the manufacturer's instructions. The double strands of small interfering RNAs (siRNAs) targeting NR4A2 (GenePharma, Shanghai, China) were as follows: siRNA (1) ( Fw: 5'-CGCGAAAUAUGUGUGUUUATT-3'; Rev：5'-UAAACACACAUAUUUCGCGTT-3' ); siRNA (2) (Fw:5'-GACCAUGUGACUUUCAAUATT-3'; Rev: 5'-UAUUGAAAGUCACA UGGUCTT-3'); siRNA (3) (Fw: 5'-GACCUCACCAACACUGAAATT-3'; Rev:5'-UUUCA GUGUUGGUGAGGUCTT-3'); Negative control (Fw:5'-UUCUCCGAACGUGUCACG UTT -3'; Rev: 5'-ACGUGACACGUUCGGAGAATT-3'). Briefly, $100 \mathrm{nmol}$ siRNAs and $5 \mu \mathrm{li}$ pofectamine 2000 were diluted in serum and antibiotic free opti-DMEM (Invitrogen) at a final volume of $500 \mu \mathrm{l}$. After mixing for $20 \mathrm{~min}$ at room temperature, the siRNA/Lipofect amine 2000 mixture was added to the cells and incubated at $37^{\circ} \mathrm{C}$ in a humidified $\mathrm{CO}_{2}$ incubator. Nonsilencing siRNA with no known homology to rat genes was synthesized as a negative control.

\section{Primary HSC isolation}

Sprague-Dawley rats (SLAC, Shanghai, China) for primary HSCs were prepared. After anesthesia, the rat abdominal wall was opened and the intestines were moved aside so as to expose the vena cava and portal vein. A fine cannula ligated and fixed to a proper location was inserted into the vein after placing a thread around it. Perfusion solutions were incubated at $37^{\circ} \mathrm{C}$ 
149 before usage. An adjustable peristaltic pump was then used to control the in situ liver perfusion

150 process. After the liver swelled, the cannula in the inferior vena cava was opened to allow

151 draining. Subsequently, the liver was perfused in turn with D-HanK's solution, Collagenase IV

152 solution, Pronase solution for $15 \mathrm{~min}$ at $37^{\circ} \mathrm{C}$. The cell fraction was collected, washed and put

153 between a top layer of buffer and a bottom cushion of 18\% Nycodenz. After centrifugation at

$1541400 \mathrm{~g}$ for 22 minutes, the HSCs fraction at the interface between the top and intermediate layer

155 was obtained. Finally, the cells were cultured in DMEM medium supplemented with 10\% FBS.

156

157 Western blotting

158 Western blot analysis was performed according to standard protocols as described previously 159 (Ogawa et al., 2012). Western blots were performed using antibodies against $\alpha$-SMA (1:500), 160 GAPDH (1:1000), NR4A1 (1:500), NR4A2 (1:500), NR4A3 (1:500), IRDye680 anti-rabbit 161 (1:5000), IRDye680 anti-mouse (1:5000), Erk1/2 (1:5000), P-erk1/2 (1:1000), JNK (1:1000), PJNK (1:1000), P-P38 (1:1000) and P38 (1:1000).

163 Protein samples were subjected to SDS-PAGE and then transferred onto a polyvinylidenefluoride membrane. After blocking, the membranes were incubated with primary antibodies followed by peroxidase-conjugated secondary antibodies. The protein samples were scanned through Odyssey Infrared Imaging System.

\section{CCK-8 assay}

169 Cell viability was assessed using the Cell Counting Kit-8 (Dojindo, KMJ, Japan) according to the 
170

171

172

173

174

175

176

177

178

179

180

181

182

183

184

185

186

187

188

189

190

manufacturer's instructions. Briefly, HSC-T6 cells transfected with NR4A2 siRNA were seeded into 96-well plates $\left(3 \times 10^{3}\right.$ cells per well). Next, $10 \mu \mathrm{l}$ of CCK8 was added to each well and the cells were incubated for $2 \mathrm{~h}$ at $37^{\circ} \mathrm{C}$. Absorbance was assessed at $450 \mathrm{~nm}$ with a microplate reader (Bio-Tek, Winooski, VT). The mean optical density (OD) of the wells in each group was used to draw cell growth curve.

\section{Cell cycle analysis}

Cells were seeded into 6 -well cell culture plates at a density of $5 \times 10^{5}$ cells. The cells were transfected with siRNA NR4A2 and 48 h later the cells were harvested, stained and fixed with propidium iodide (PI) according to the manufacturer's protocol and subject to cell cycle analysis using flow cytometer (BD, NK, USA).

\section{Cell apoptotic assay}

Cells were seeded into 6 -well cell culture plates at a density of $5 \times 10^{5}$ cells. The cells were transfected with siRNA NR4A2 and 48 hours later the cells were harvested. Apoptosis assay was measured using FITC AnnexinV Apoptosis Detection Kit according to the manufacturer's protocol and subject to cell cycle analysis using flow cytometer.

Statistical analysis

The results were expressed as mean \pm SD and were analyzed by analysis of variance (ANOVA) or Student's $t$-test followed by paired comparison as appropriate. $\mathrm{P}<0.05$ was taken as the minimum 
191

192

193

194

195

196

197

198

199

200

201

202

203

204

205

206

207

208

209

210

level of significance.

\section{RESULTS}

\section{NR4A2 expression was reduced in activated HSCs}

HSCs play an important role in liver fibrosis. Quiet HSCs could be activated by numerous factors and produce extracellular matrix. $\alpha$-SMA is a well-established marker for liver fibrosis. PDGF and TGF- $\beta$ are strong stimulating factors. After HSC-T6 cells was stimulated by PDGF BB (10 ng/ml or $20 \mathrm{ng} / \mathrm{ml}), \mathrm{NR} 4 \mathrm{~A} 2 \mathrm{mRNA}$ level decreased significantly whereas $\alpha$-SMA level increased significantly (Fig. 1A). The level of NR4A2 protein has significantly reduced after treatment with PDGF BB (10ng/ml, 20ng/ml respectively) in HSC-T6 cells, yet inconspicuous changes occurred in NR4A1 and NR4A3 expression (Fig. 1B). Similar observation has been made after treatment with TGF- $\beta$ (2ng/ml) (Fig. 1C). We also screened NR4A2 expression on short time upon stimulation by PDGF BB (Fig.1D) or TGF- $\beta$ (Fig. 1E). It was revealed NR4A2 mRNA level for $12 \mathrm{~h}$ increased significantly compared to control group meanwhile NR4A2 protein levels elevated slightly after stimulated by PDGF BB (Fig. 1F) or TGF- $\beta$ (Fig. 1G).

Further, we isolated primary HSCs from SD rats. Primary hepatic stellate cells can be spontaneously stimulated and proliferate several days after isolation (Davis et al., 1988). We observed that NR4A2 mRNA expression decreased significantly while $\alpha$-SMA level was increased 2-7-folds in primary hepatic stellate cells after isolation (Fig. 2). 
212

213

214

215

216

217

218

219

220

221

222

223

224

225

226

227

a -SMA expressed mainly in fibrotic area where extracellular matrix accumulated whilst NR4A2 expressed in both fibrotic and non-fibrotic area of liver tissue. However, fibrotic area was dominant in fibrotic liver compared with normal liver. Immunohistochemistry analysis illustrated lower expression of NR4A2 in fibrotic area than non-fibrotic area (Fig.3A, B). The same result was observed by immunofluorescence analysis (Fig. 3C).

\section{NR4A2 inhibited $\alpha$-SMA and Col1 expression in HSCs}

To clarify the correlation between NR4A2 and liver fibrosis, we knocked down NR4A2 via three siRNAs and examined the expression of extracellular matrix markers in HSC-T6 cells. RT-PCR analysis showed that the mRNA level of NR4A2 decreased by more than $50 \%$ (Fig. 4A) while $\alpha$-SMA and Col1 increased by more than 50\% ( Fig. 4B, C).

\section{NR4A2 suppressed cell proliferation, modulated cell cycle and promoted apoptosis}

After NR4A2 knocked down in HSC-T6 cells, we analyzed cell cycle and apoptosis by flow cytometry and examined optical density by CCK8 assay. Flow cytometry showed decreased percentage of cells in G1 phase and increased percentage in S stage compared with control group after NR4A2 knockout (Fig. 5A, B). NR4A2 knockout also led to reduced apoptosis (Fig. 5C, D) and promoted proliferation (Fig. 5E) in HSC-T6 cells.

\section{NR4A2 upregulation of MAPK pathway in HSC proliferation}

In liver fibrogenesis, MAPK is an important signaling pathway which consists of ERK1/2, P38 and JNK. Western blotting analysis indicated reduced phosphorylation of ERK1/2, P38 and JNK with NR4A2 knockout in HSC-T6 cells (Fig. 6A-C). 


\section{DISCUSSION \&CONCLUSIONS}

234 Liver fibrosis is prevalent worldwide and in which HSCs play a central role (Ueno et al., 1997).

235 On one hand, clearance of activated HSCs by apoptosis remained appealing for antifibrotic

therapy; on the other hand, activated HSCs could also revert to quiescent phenotype (Olaso et al., 2001; Gaça et al., 2003; Guyot et al., 2007). The mechanism by which activated HSCs are suppressed in liver fibrosis deserves to be extensively investigated.

Accumulated evidence suggested nuclear receptors mediate key steps in activation of HSCs

(Marra et al., 2000; Hellemans et al., 2004; Milliano \& Luxon., 2005). Peroxisome proliferator

activated receptor- $\gamma$ (PPAR- $\gamma$ ) inhibits pro-fibrogenic genes expression in quiescent HSCs.

Treatment with PPAR- $\gamma$ agonists restrains HSCs from activation and collagen production (Galli

et al., 2002; Marra et al., 2005; Chen et al., 2008; Yang et al., 2010). RAR and RXR exhibit

similar effect on HSCs (Marra et al., 2000; Qin et al., 2008). NR4A1 exhibited lower expression

in liver fibrosis. Long-time stimulation with TGF- $\beta$ down-regulates NR4A1 level while short-

time stimulation leads to increased NR4A1 in human dermal fibroblasts (Palumbo-Zerr et al.,

2015). Yin et al investigated the role of NR4A members in uterine fibroids. He found expression

of NA4A1, NR4A2 and NR4A3 are remarkably lower in leiomyoma than control. The

reductions in NA4A2 and NR4A3 are significantly higher than NR4A1 in human fibroid (Yin et

al., 2013). In agreement with above results, our study indicated that NR4A2 expression

decreased in liver fibrosis and PDGF BB or TGF- $\beta$ activated HSCs. At the same time no

significant change was observed in NA4A1 or NR4A3. NR4A2 is mainly involving in functional 
254 Alzheimer's disease and Parkinson's disease (Baron et al., 2012; Park et al., 2012; Aldavert-

255 Vera et al., 2013; Carloni et al., 2013; Moon et al., 2014). To the best of our knowledge, this is

256 the first report on the effect of NR4A2 in liver fibrosis. To further test the effect of short-time

257 stimulation, we assessed NR4A2 level for less than 24 hours in HSC-T6 cells after stimulation.

258 In response to PDGF BB, NR4A2 mRNA levels elevated from $1 \mathrm{~h}$ to $24 \mathrm{~h}$ and in particular its

259 level for $12 \mathrm{~h}$ was increased more than 60-fold. In contrast, NR4A2 protein levels did not

260 increased significantly from $3 \mathrm{~h}$ to $24 \mathrm{~h}$. The similar results were obtained upon stimulation with

261 TGF- $\beta$. To some extent, our results are consistent with Palumbo-Zerr et al.

262 Primary hepatic stellate cells can be spontaneously stimulated and proliferate several days

263 after isolation meanwhile quiet HSCs transform into activated HSCs which resembles the

264 process in vivo (Davis et al., 1988). We found sharp decrease of NR4A2 expression and obvious

265 increase in a -SMA and Coll expression in isolated hepatic stellate cells. Through NR4A2

266 knockout, a -SMA and Col1, another characteristic marker for liver fibrosis down-regulated

267 dramatically. Besides, we found that overexpression of NR4A2 led to reduced a -SMA level in

268 both HSC-T6 cells and primary hepatic stellate cells (data not shown). Taken together, there is a

269 correlation between NR4A2 and liver fibrosis and NR4A2 could down-regulate ECM genes.

270 NR4A2 expression in prostate tissue is conspicuously elevated and with NR4A2 knockout

271 the cell proliferation, infiltration and migration are inhibited (Wang et al., 2013). Different from

272 cancer, we found that loss of NR4A2 induced reduced apoptosis, promoted proliferation,

273 decreased percentage of cells in G1 phase and increased percentage in S stage in liver fibrosis.

274 On the contrary, overexpression of NR4A2 reduces cell proliferation (Yin et al., 2013). 
NR4A2 can be mediated by a variety of signaling pathways (Barish et al., 2005) MAPK

276

277

278

pathway is prominent among them. MAPK consists of ERK1/2, P38 and JNK. Our study showed that NR4A2 silencing repressed phosphorylation of ERK1/2, P38 and JNK. Inversely, overexpression of NR4A2 induced phosphorylation of ERK1/2, P38 and JNK (data not shown). It may be concluded that NR4A2 suppresses cell proliferation via phosphorylating ERK1/2, P38 and JNK tin HSCs. Treatment with TGF- $\beta$ resulted in decreased NR4A2 level and knockout of NR4A2 induced reduced phosphorylation of ERK1/2, P38 and JNK. So it seems that stimulation with TGF- $\beta$ lead to suppressed phosphorylation of MAPK. This is paradoxical with the theory that TGF- $\beta$ induces MAPK/ERK activation in liver fibrogenesis (Reimann et al., 1997). Yet, it was confirmed that via inhibition of ERK activation TGF- $\beta$ represses cell proliferation in pancreatic carcinoma cells and T cells (Giehl et al., 2000; Luo et al., 2008). We speculate that NR4A2 is not the sole target of TGF- $\beta$ and other genes may counteract NR4A2. Moreover, TGF$\beta$ and NR4A2 may interact with each other. But the mechanism is unknown at present. Meanwhile, we also noticed high expression of NR4A2 in hepatocytes in liver tissue. It is not contradictory as other cells express NR4A2 in vivo. Taura et al demonstrated that hepatocytes are not involved in liver fibrogenesis (Taura K et al., 2010).

Our study demonstrated NR4A2 down-regulate HSCs proliferation through MAPK pathway and reduce extracellular matrix accumulation in liver fibrogenesis. However there are some drawbacks such as lack of animal experiments. The target gene of NR4A2 and the mechanism by which NR4A2 regulate HSCs need to be explored in future study. In summary, NR4A2 can inhibit proliferation of HSC through regulation of MAPK pathway and reduce extracellular 
296

297

298

299

300

301

302

303

304

305

306

307

308

matrix. NR4A2 may be a promising target for anti-fibrosis.

\section{REFERENNCES}

\section{Aldavert-Vera L, Huguet G, Costa-Miserachs D, Ortiz SP, Kádár E, Morgado-Bernal I, Segura-Torres} P. 2013. Intracranial self-stimulation facilitates active-avoidance retention and induces expression of cFos and Nurr1 in rat brain memory systems. Behav Brain Res 250: 46-57.

Barish GD, Downes M, Alaynick WA, Yu RT, Ocampo CB, Bookout AL, Mangelsdorf DJ, Evans RM. 2005. A Nuclear Receptor Atlas: macrophage activation. Mol Endocrinol 19: 2466-77.

\section{Baron O, Förthmann B, Lee YW, Terranova C, Ratzka A, Stachowiak EK, Grothe C, Claus P,} Stachowiak MK. 2012. Cooperation of nuclear fibroblast growth factor receptor 1 and Nurr1 off ers new interactive mechanism in postmitotic development of mesencephalic dopaminergic neurons. J Biol Chem 287:19827-19840.

Cao Q, Mak KM, Ren C, Lieber CS. 2004. Leptin stimulates tissue inhibitor of metalloproteinase-1 in human hepatic stellate cells: respective roles of the JAK/STAT and JAK-mediated H2O2-depen dant MAPK pathways. J.Biol.Chem 279: 4292-4304.

Carloni M, Nasuti C, Fedeli D, Montani M, Vadhana MS, Amici A, Gabbianelli R. 2013. Early life permethrin exposure induces long-term brain changes in Nurr1, NF-kB and Nrf-2. Brain Res 1515: 19-28.

Cassiman D, Libbrecht L, Desmet V, Denef C, Roskams T. 2002. Hepatic stellate cell/myofibroblas $\mathrm{t}$ subpopulations in fibrotic human and rat livers. $J$ Hepatol 36: 200-9.

Chen H, He YW, Liu WQ, Zhang JH. 2008. Rosiglitazone prevents murine Hepatic fibrosis induced by Schistosomajaponicum. World J Gastroenterol 14: 2905-2911. 
317

318

319

320

321

322

323

324

325

326

327

328

329

330

331

332

333

334

335

336

337

Davis BH, Vucic A. 1988. The effect of retinol on Ito cell proliferation in vitro. Hepatology 8: 788-793.

Gaça MD, Zhou X, Issa R, Kiriella K, Iredale JP, Benyon RC. 2003. Baseme membrane-like matrix inhibits proliferation and collagen synthesis by activated rat hepatic stellate cells: evidence for matrixdependent deactivation of stellate cells. Matrix Biol 22: 229-239.

Galli A, Crabb DW, Ceni E, Salzano R, Mello T, Svegliati-Baroni G, RidolfiFTrozzi L, Surrenti C, Casini A. 2002. Antidiabetic thiazolidinediones Inhibit collagen synthesis and hepatic stellate cell activation in vivo and in vitro. Gastroenterology 122: 1924-1940.

García-Pérez D, Sáez-Belmonte F, Laorden ML, Núñez C, Milanés MV. 2013. Morphine administration modulates Expression of Argonaute2 and dopamine-related transcription Factors involved in midbrain dopaminergic neurons function. Brit J Pharmacol 168: 1889-1901.

García-Pérez D, López-Bellido R, Hidalgo JM, Rodríguez RE, Laorden ML, Núñez C, Milanés MV. 2015. Morphine regulates Argonaute 2 and $\mathrm{TH}$ expression and activity but not miR-133b in Midbrain dopaminergic neurons. Addict Biol 20: 104-19.

Giehl K, Seidel B, Gierschik P, Adler G, Menke A. 2000. TGF beta1 represses proliferation of pancreatic carcinoma cells which correlates with Smad4-independent inhibition of ERK activation. Oncogene 19: 4531-41.

Guyot C, Combe C, Balabaud C, Bioulac-Sage P, Desmoulière A. 2007. Fibrogenic cell fate during fibrotic tissue remodelling observed in rat and humancultured liver slices. J Hepatol 46: 142-150.

Hellemans K, Verbuyst P, Quartier E, Schuit F, Rombouts K, Chandraratna RA,Schuppan D, Geerts A. 2004. Differential modulation of rat heaptic stellate phenotype by natural and synthetic retinoids. Hepatology 39: 97-108. 
Holla VR, Wu H, Shi Q, Menter DG, DuBois RN. 2011. Nuclear orphan receptor NR4A2 modulates fatty acid oxidation pathways in colorectal cancer. $J$ Biol Chem 286: 30003-9.

Luo X, Zhang Q, Liu V, Xia Z, Pothoven KL, Lee C. 2008. Cutting edge: TGF-beta-induced expression of Foxp3 in T cells is mediated through inactivation of ERK. J Immunol 180: 2757-61.

Marra F, DeFranco R, Robino G, Novo E, Efsen E, Pastacaldi S, Zamara E, Vercelli A, Lottini B, Spirli C, Strazzabosco M, Pinzani M, Parola M. 2005. Thiazolidinedione treatment Inhibits bile duct proliferation and fibrosisin a rat model of Chronic cholestasis. World J Gastroenterol 11: 4931-4938.

Marra F, Efsen E, Romanelli RG, Caligiuri A, Pastacaldi S, Batignani G, Bonacchi A, Caporale R, Laffi G, Pinzani M, Gentilini P. 2000. Ligands of peroxisome proliferator-activated receptor gamma modulate profibrogenic and proinflammatory actions in hepatic stellate cells .Gastroenterology 119: 466-478.

MillianoMT, LuxonBA. 2005. Rat hepatic stellate cells become retinoid unresponsive during activation. Hepatol Res 33: 225-233.

Moon M, Jeong I, Kim CH, Kim J, Lee PK, Mook-Jung I, Leblanc P, Kim KS. 2015. Correlation between orphan nuclear receptor Nurr1 expression and amyloid deposition in 5XFAD mice, an animal model of Alzheimer's disease. $J$ Neurochem 132: 254-262.

Ogawa T, Enomoto M, Fujii H, Sekiya Y, Yoshizato K, Ikeda K, Kawada N. 2012. MicroRNA-221/222 upregulation indicates the activation of stellate cells and the progression of liver fibrosis. Gut 61: 1600-9.

Olaso E, Ikeda K, Eng FJ, Xu L, Wang LH, Lin HC, Friedman SL. 2001. DDR2 receptor promotes MMP2-mediated proliferation and invasion by hepatic stellate cells. J Clin Invest 108: 1369-1378.

Palumbo-Zerr K, Zerr P, Distler A, Fliehr J, Mancuso R, Huang J, Mielenz D, Tomcik M, Fürnrohr BG, Scholtysek C, Dees C, Beyer C, Krönke G, MetzgerD, Distler O, Sc-hett G, Distler JH. 2015. Orphan 
359

360

361

362

363

364

365

366

367

368

369

370

371

372

373

374

375

376

377

378

379

nuclear receptor NR4A1 regulates transforming growth factor- $\beta$ signaling and fibrosis. Nat Med 21: $150-158$

Park JS, Yang HN, Woo DG, Jeon SY, Do HJ, Huh SH, Kim NH, Kim JH, Park KH. 2012. Exogenous Nurr1 gene expression in electrically-stimulated human MSCs and the induction of neurogenesis. Biomaterials 33: 7300-7308.

Qin X, Xie X, Fan Y, Tian J, Guan Y, Wang X, Zhu Y, Wang N. 2008. Peroxisome proliferator-activated receptor-delta induces insulin-induced gene-1 and suppresses hepatic lipogenesis in obese diabetic mice. Hepatology 48: 432-441.

Reimann T, Hempel U, Krautwald S, Axmann A, Scheibe R, Seidel D, Wenzel KW. 1997. Transforming growth factor-beta1 induces activation of Ras, Raf-1, MEK and MAPK in rat hepatic stellate cells. FEBS Lett 403: 57-60.

Reynaert H, Thompson MG, Thomas T, Geerts A. 2002. Hepatic stellate cells: role in microcirculation and pathophysiology of portal hypertension. Gut 50: $571-81$.

Rius J, Martínez-González J, Crespo J, Badimon L. 2006. NOR-1 is involved in VEGF-induced endothelial cell growth. Atherosclerosis 184: 276-82.

Sacchetti P, Carpentier R, Ségard P, Olivé-Cren C, Lefebvre P. 2006. Multiple signaling pathways regulate the transcriptional activity of the orphan nuclear receptor Nurr1. Nucleic Acids Res 34: 55155527.

Saxena NK, Sharma D, Ding X, Lin S, Marra F, Merlin D, Anania FA. 2007. Concomitant activation of the JAK/STAT, PI3K/AKT, and ERK signaling is involved in leptin-mediated promotion of invasion and migration of hepatocellular carcinoma cells. Cancer Res 67: 2497-507. 
Taura K1, Miura K, Iwaisako K, Osterreicher CH, Kodama Y, Penz-Osterreicher M, Brenner D. 2010. Hepatocytes do not undergo epithelial-mesenchymal transition in liver fibrosis in mice. Hepatology 51: 1027-1036.

Ueno T, Sata M, Sakata R, Torimura T, Sakamoto M, Sugawara H, Tanikawa K. 1997. Hepatic stellate cells and intralobular innervation in human liver cirrhosis. Hum Pathol 28: 953-9.

Wang J, Yang J, Zou Y, Huang GL, He ZW. 2013. Orphan nuclear receptor nurr1 as a potential novel marker for progression in human prostate cancer. Asian Pac J Cancer Prev14: 2023-8.

Yang L, Stimpson SA, Chen L, Wallace Harrington W, Rockey DC. 2010. Effectiveness of the PPARgamma agonist, GW570, in liver fibrosis. Inflamm Res 59: 1061-1071.

Yin H, Lo JH, Kim JY, Marsh EE, Kim JJ, Ghosh AK, Bulun S, Chakravarti D. 2013. Expression profiling of nuclear receptors identifies key roles of NR4A subfamily in uterine fibroids. Mol Endocrinol 27: 726-40.

Zhang T, Jia N, Fei E, Wang P, Liao Z, Ding L, Yan M, Nukina N, Zhou J, Wang G. 2007. Nurr1 is phosphorylated by ERK2 in vitro and its phosphorylation Upregulates tyrosine hydroxylase expression in SH-SY5Ycells. Neurosci Lett 423: 118-122.

Zhao D, Desai S, Zeng H. 2011.VEGF stimulates PKD-mediated CREB-dependent orphan nuclear receptor Nurr1 expression: role in VEGF-induced angiogenesis. Int J Cancer 128: 2602-12.

Zhong W, Shen WF, Ning BF, Hu PF, Lin Y, Yue HY, Yin C, Hou JL, Chen YX, Zhang JP, Zhang X, Xie WF. 2009. Inhibition of extracellular signal-regulated kinase 1 by adenovirus mediated small interfering RNA attenuates hepatic fibrosis in rats. Hepatology 50: 1524-1536. 


\section{1}

Expression of NR4A1, NR4A2, NR4A3 and $\alpha$-SMA in HSC-T6 cells.

(A) Expression of NR4A1, NR4A2, NR4A3 and $\alpha$-SMA in HSC-T6 cells stimulated with or without PDGF BB $(20 \mathrm{ng} / \mathrm{ml})$ for $48 \mathrm{~h}$ were examined by real-time PCR $(\mathrm{n} \geq 3)$. $* \mathrm{P}<0.05 \mathrm{vs}$. NC.

(B) Western blot analysis of NR4A1, NR4A2 and NR4A3 in HSC-T6 cells stimulated by PDGF $B B$ in different concentrations $(0 \mathrm{ng} / \mathrm{ml}, 10 \mathrm{ng} / \mathrm{ml}$ and $20 \mathrm{ng} / \mathrm{ml}$ ) for $48 \mathrm{~h}$. (C) Western blot analysis of NR4A1, NR4A2 and NR4A3 in HSC-T6 cells stimulated with or without TGF- $B(2$ $\mathrm{ng} / \mathrm{ml}$ ) for $48 \mathrm{~h}$. (D) Real-time PCR analysis of NR4A2 levels in HSC-T6 cells stimulated by PDGF BB $(20 \mathrm{ng} / \mathrm{ml})$ for $1 \mathrm{~h}, 12 \mathrm{~h}$ and $24 \mathrm{~h}(\mathrm{n} \geq 3)$. ***P<0.001 vs. NC. (E) Real-time PCR analysis of NR4A2 levels in HSC-T6 cells stimulated by TGF- $\beta(2 \mathrm{ng} / \mathrm{ml})$ for $1 \mathrm{~h}, 12 \mathrm{~h}$ and $24 \mathrm{~h}$ $(n \geq 3)$. ${ }^{* * *}<0.001$ vs. NC. (F) Western blot analysis of NR4A2 levels in HSC-T6 cells stimulated by PDGF BB $(20 \mathrm{ng} / \mathrm{ml})$ for $3 \mathrm{~h}, 12 \mathrm{~h}$ and $24 \mathrm{~h}$. (G) Western blot analysis of NR4A2 levels in HSC-T6 cells stimulated by TGF- $\beta$ ( $2 \mathrm{ng} / \mathrm{ml})$ for $3 \mathrm{~h}, 12 \mathrm{~h}$ and $24 \mathrm{~h}$. 

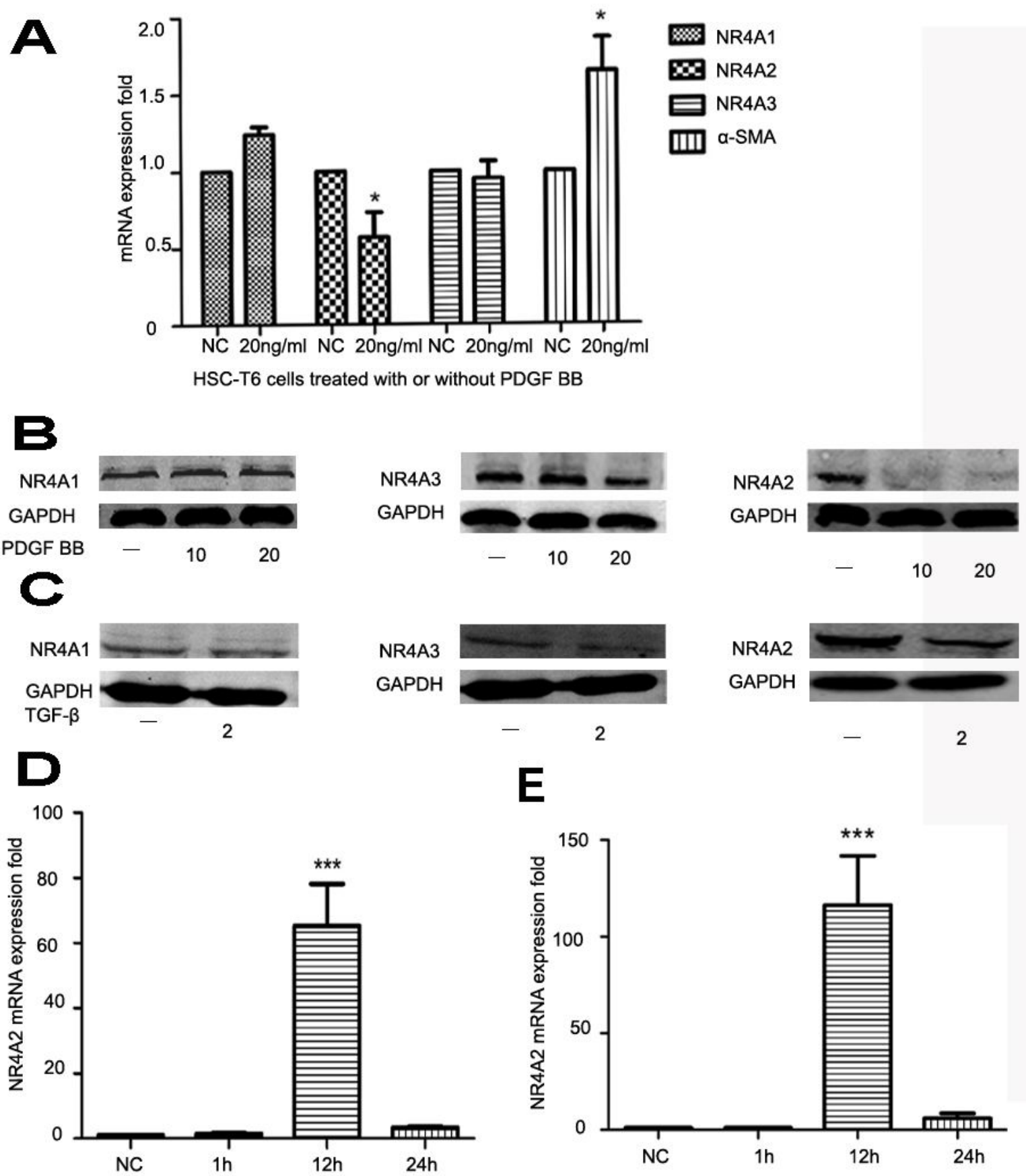

E
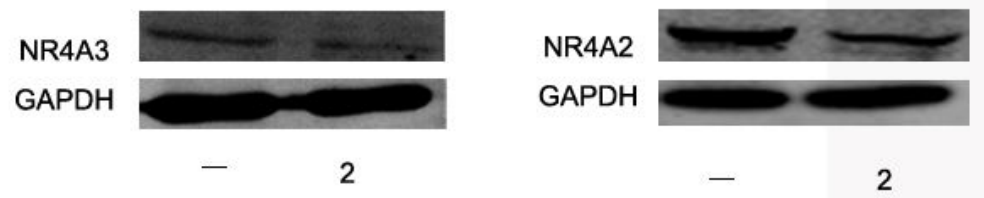

HSC-T6 cells tteated with PDGF BB

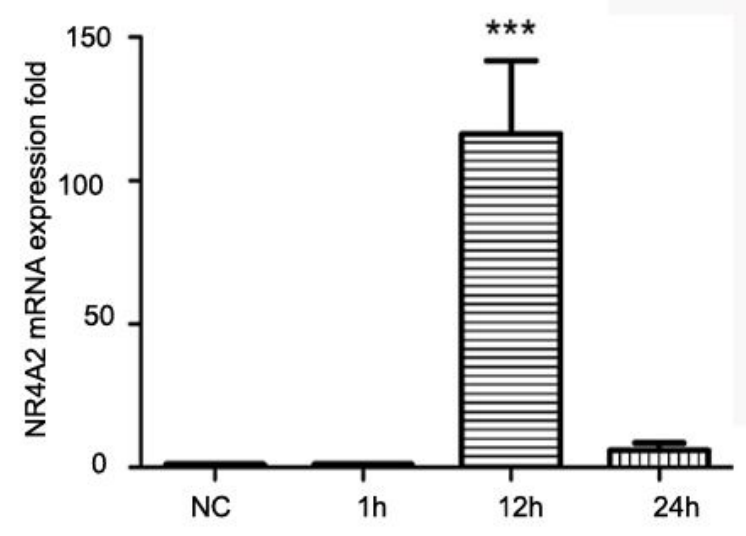

HSC-T6 cells treated with TGF- $\beta$

$\mathbf{F}$

G
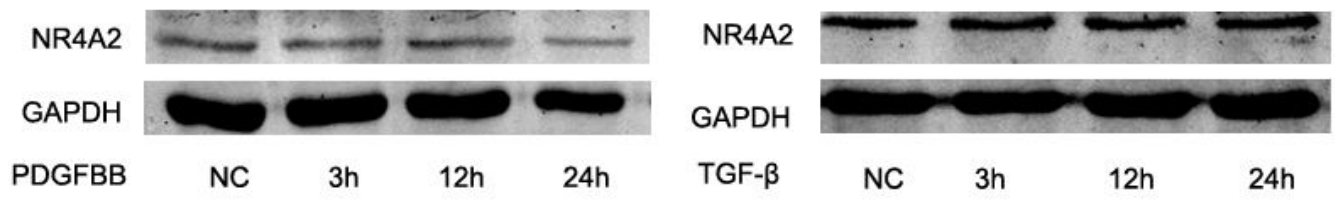

TGF- $\beta \quad$ NC $\quad 3 h$

$12 \mathrm{~h}$

$24 \mathrm{~h}$ 
2

Expression of NR4A2 and $\alpha$-SMA in primary hepatic stellate cells

Expression of NR4A2 and $\alpha$-SMA in primary hepatic stellate cells after isolation were examined by real-time PCR $(n \geq 3)$. **P $<0.01$ vs. 0th day group. Results are expressed as mean $\pm S D$.

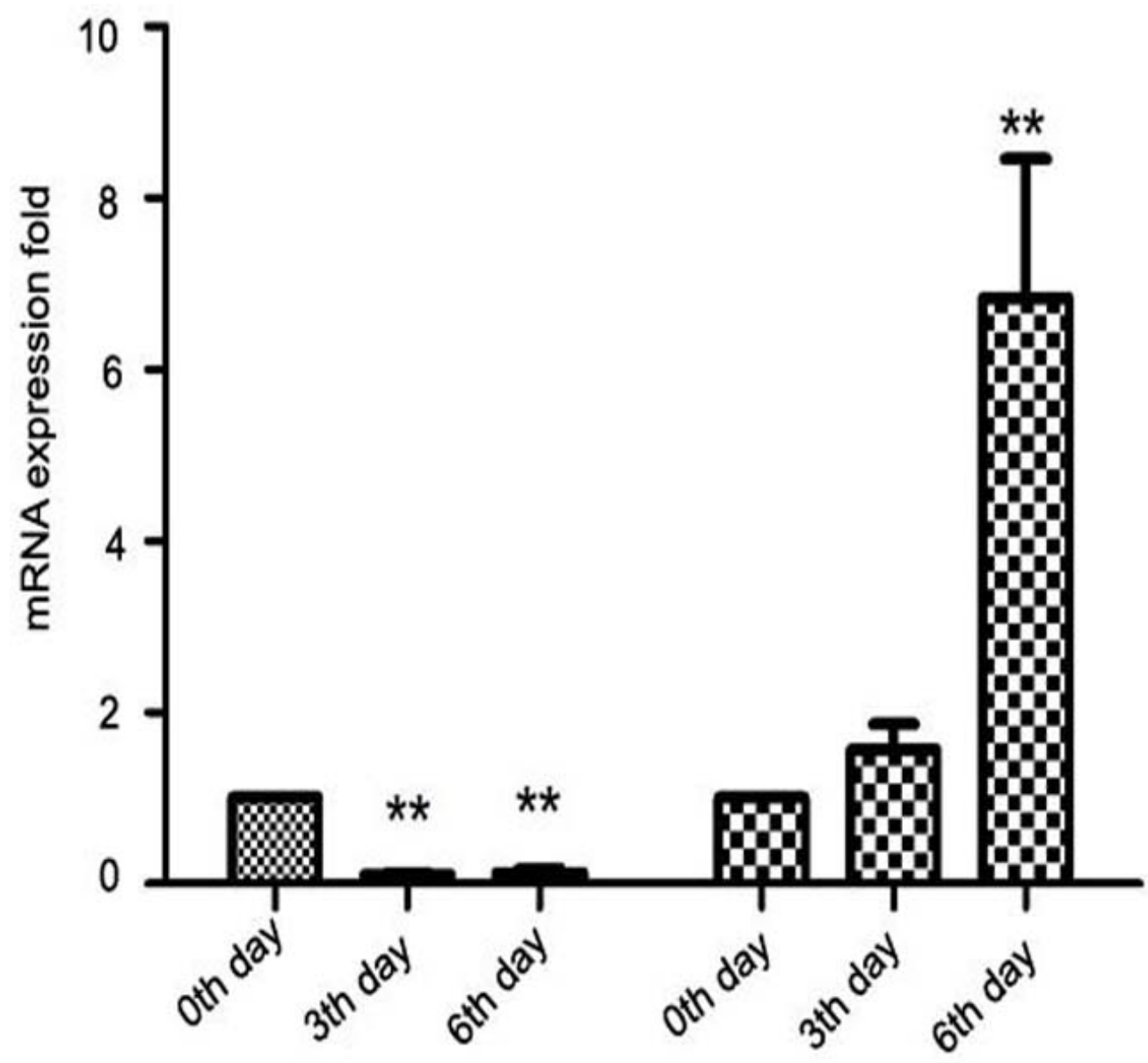

NR4A2

M. a SMA 


\section{3}

NR4A2 expression in human liver tissues.

NR4A2 expression was detected in normal liver (A) and cirrhotic liver (B) by Immunohistochemistry. (C)NR4A2 expression in human normal and cirrhosis liver tissue was confirmed by double staining with anti-NR4A2 (green) and anti- $\alpha$ SMA (red) antibodies. DAPI staining (blue) and the merged image are shown. scale bar $200 \mu \mathrm{m}$

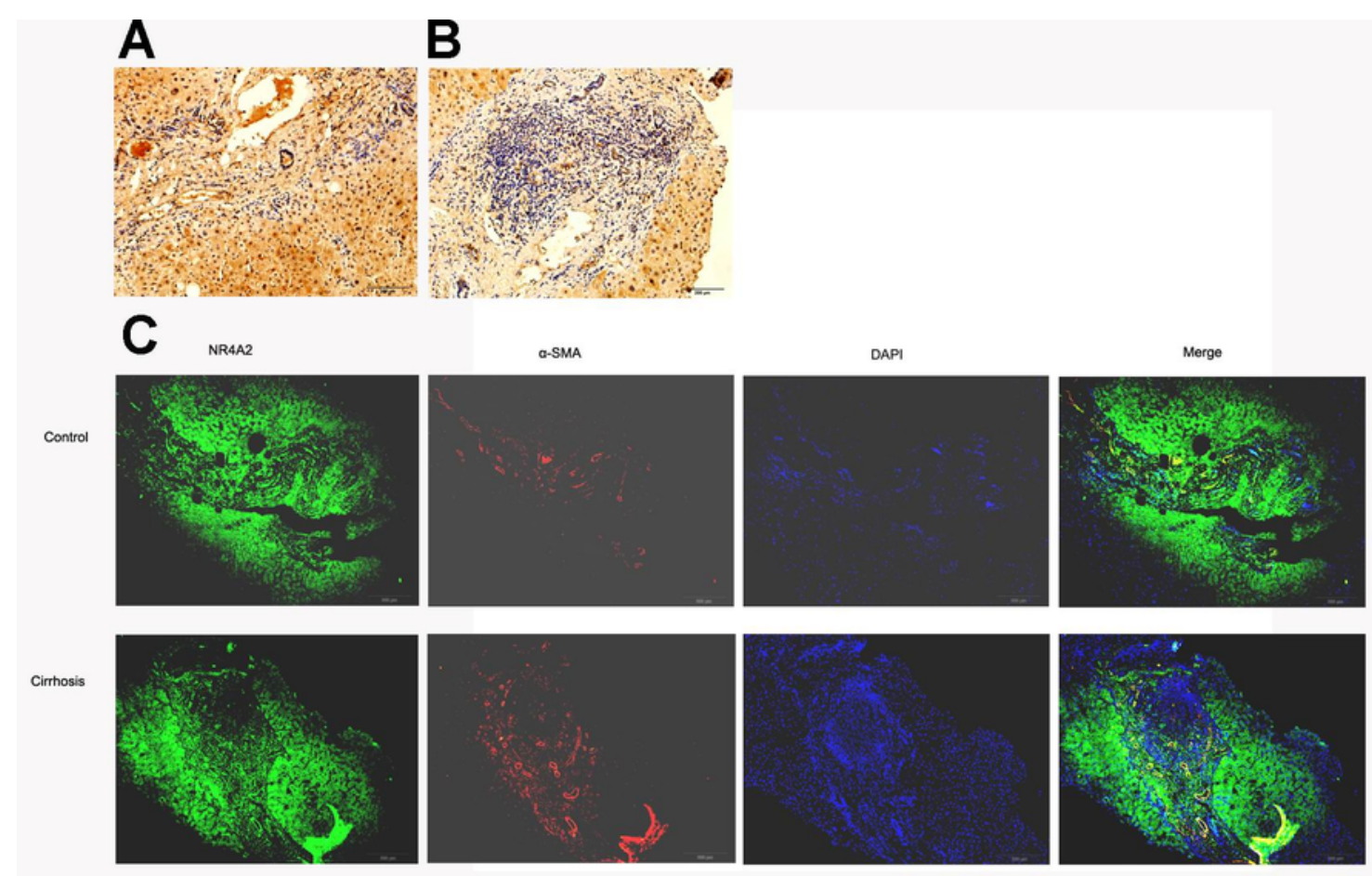


4

Analysis of NR4A2, $\alpha$-SMA and Col1 after NR4A2 knockdown in HSC-T6 cells.

Expression of NR4A2 (A), $\alpha$-SMA (B) and Col1(C) in HSC-T6 were examined by real-time PCR $(n \geq 3) . * P<0.05$ vs. NC and $* * P<0.01$ vs. NC. Results are expressed as mean $\pm S D$.

A

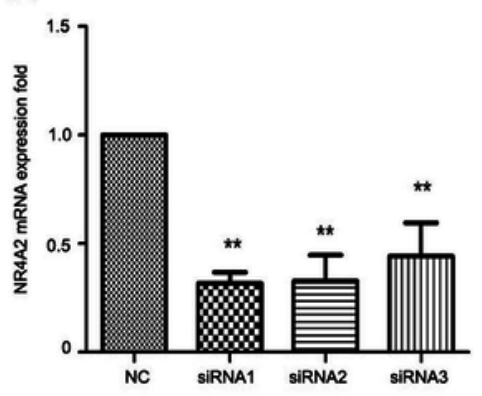

B

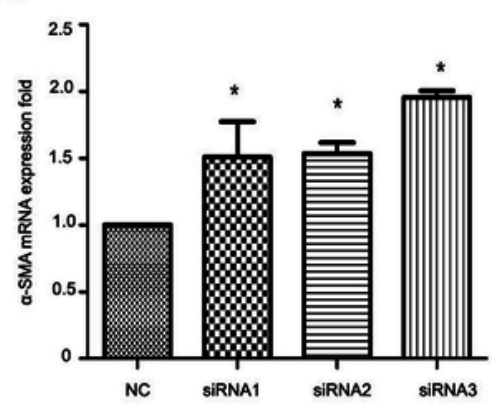

C

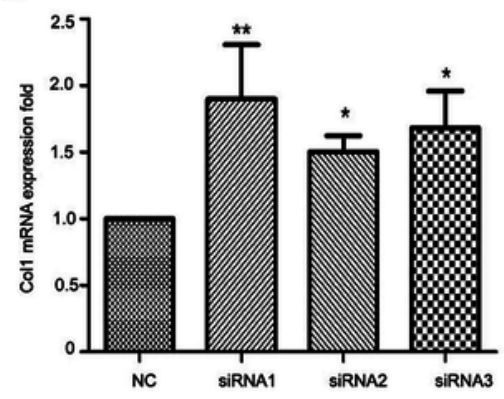




\section{5}

Analysis of cell proliferation, cell cycle and cell apoptosis after NR4A2 knockdown in HSC-T6 cells.

Analysis of cell proliferation, cell cycle and cell apoptosis after NR4A2 knockdown in HSC-T6 cells. (A, B) Analysis of cell cycle. Flow cytometry graph for control and siRNA-infected HSCT6 cells (A). Summary of cell cycle (B). The percentage of cells in G1 phase was decreased after NR4A2 knockout and the percentage of cells in S phase was increased $(n \geq 3)$. $* P<0.05$ vs. NC and ${ }^{*} * \mathrm{P}<0.01$ vs. NC. (C, D) Analysis of cell apoptosis. Flow cytometry graph for control and siRNA-infected HSC-T6 cells (C). (D) Summary of cell apoptosis. The apoptosis percentage of HSC-T6 cells after NR4A2 knockdown was remarkably decreased $(n \geq 3)$. $* P<0.05$ vs.NC. (E) Cell proliferation analyzed by cell counting kit-8 $(n \geq 3) . * P<0.05$ vs NC. Results are expressed as mean \pm SD. 
A
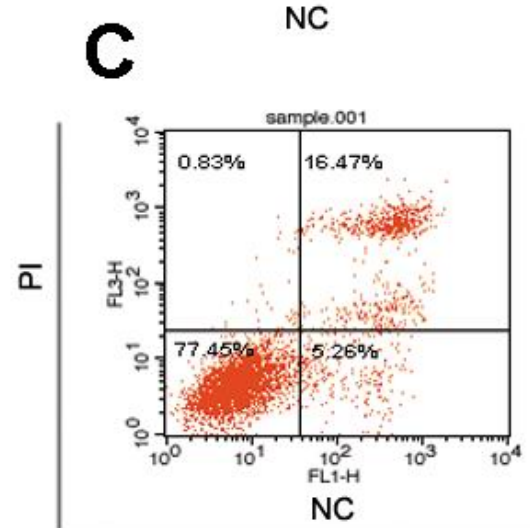

Annexin V-FITC

E

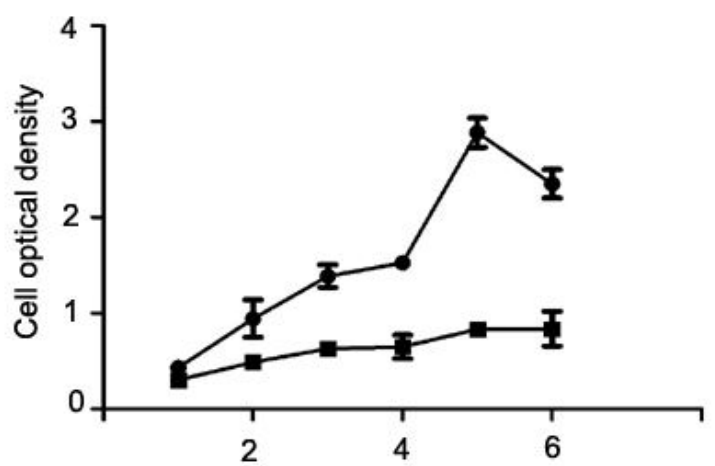

Time (day) after cells treated with siRNA
B

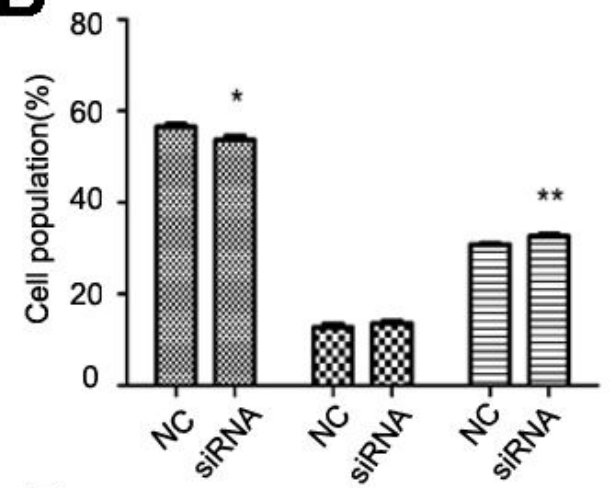

D
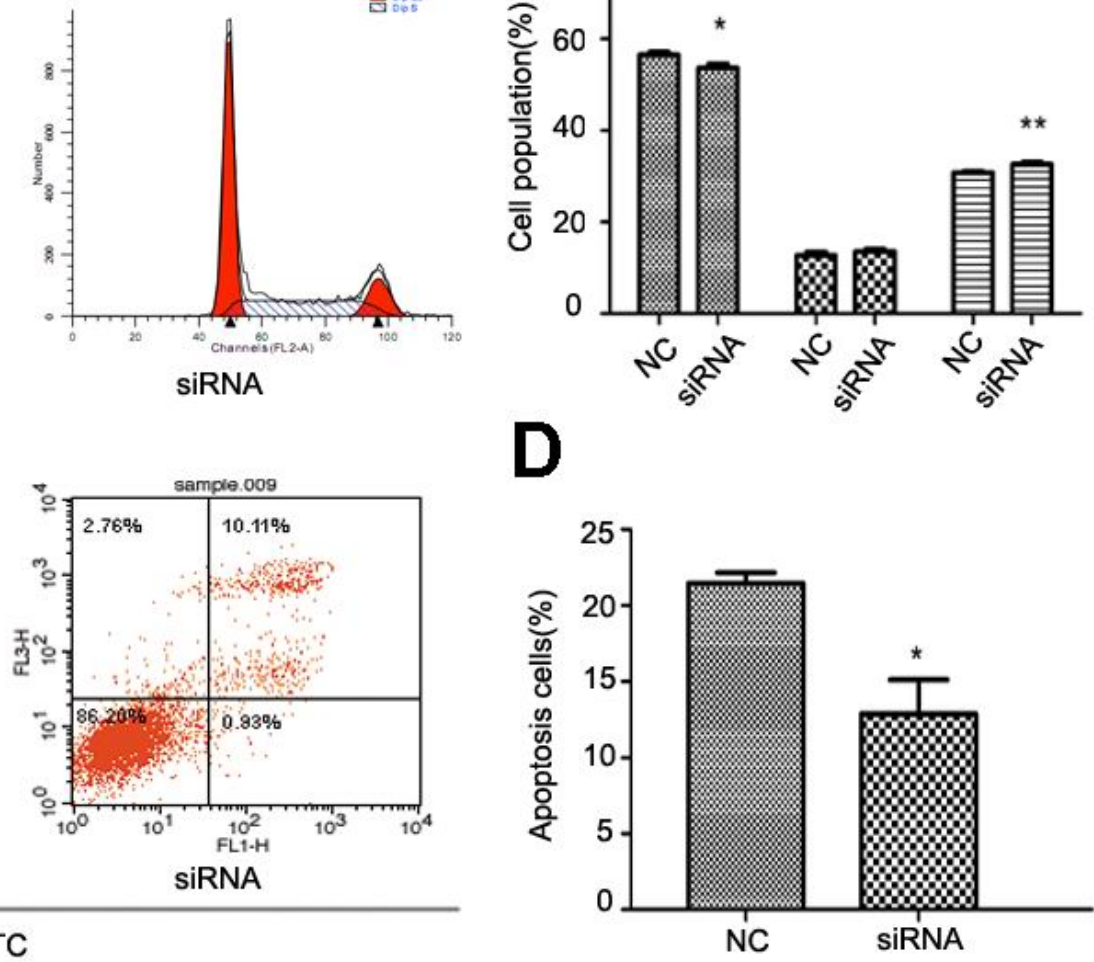

․․․

$\mathbb{G} 2$

曰 
6

Analysis of ERK1/2, P38, and JNK after NR4A2 silencing knockdown in HSC-T6 cells.

(A) Analysis of phosphorylation of ERK1/2 by Western blotting and relative intensity (P-

ERK1/2 normalized against ERK1/2) $(n \geq 3) . * P<0.05$ vs.Con. (B) Analysis of phosphorylation of JNK by Western blotting and relative intensity (P-JNK normalized against JNK) $(n \geq 3)$. $* P<0.05$ vs.Con. (C) Analysis of phosphorylation of P38 by Western blotting and relative intensity (PP38 normalized against P38) $(n \geq 3)$. **P<0.01 vs. Con. Results are expressed as mean $\pm S D$.
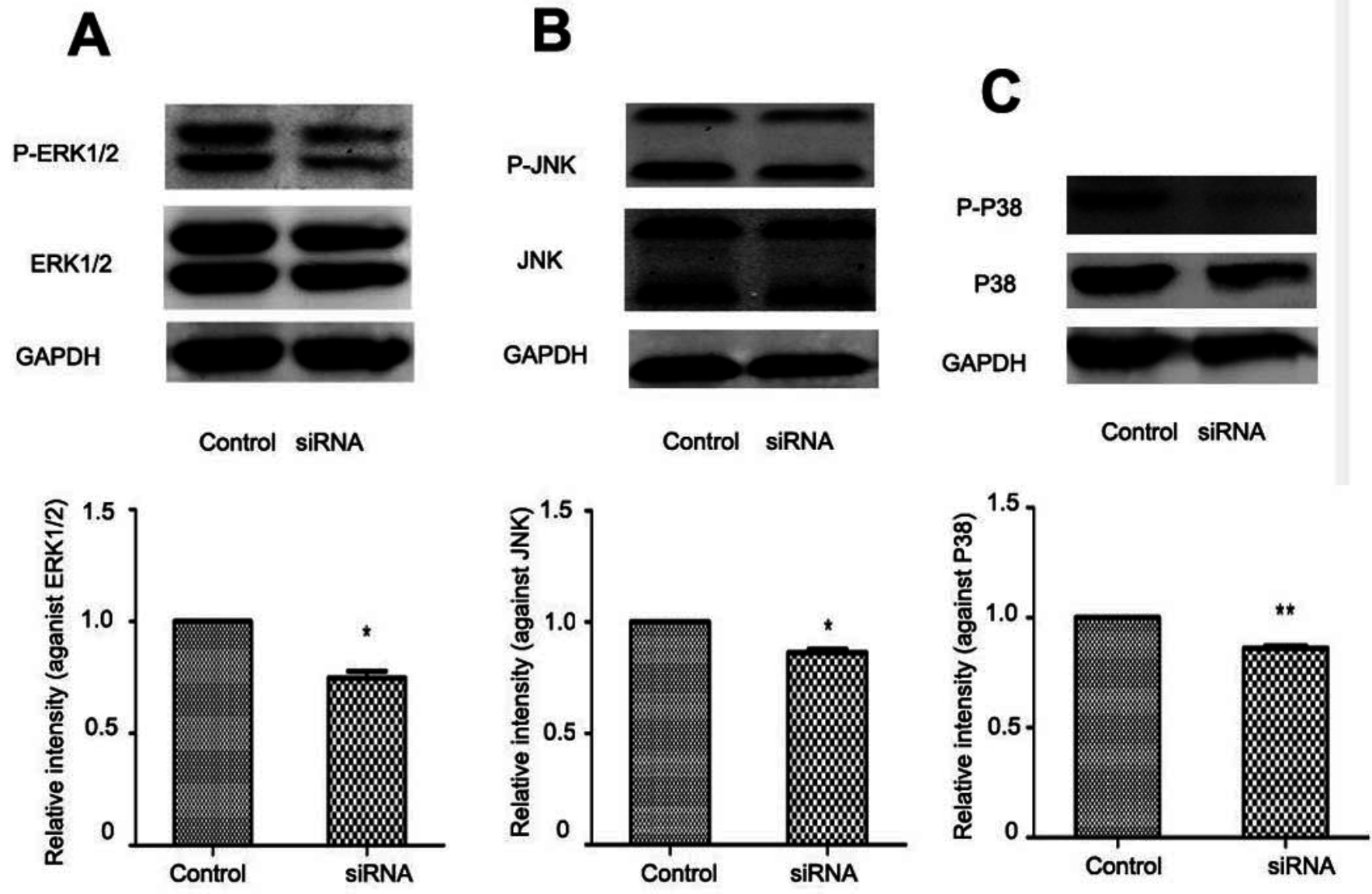